\title{
Frequent concerted genetic mechanisms disrupt multiple components of the NRF2 inhibitor KEAP1/CUL3/RBX1 E3-ubiquitin ligase complex in thyroid cancer
}

\author{
Victor D Martinez ${ }^{*}$, Emily A Vucic ${ }^{\dagger}$, Larissa A Pikor, Kelsie L Thu, Roland Hubaux and Wan L Lam
}

\begin{abstract}
Background: Reactive oxygen species contribute to normal thyroid function. The NRF2 oxidative response pathway is frequently and constitutively activated in multiple tumor types, including papillary thyroid carcinoma (PTC). Genetic mechanisms underlying NRF2 pathway activation in PTC are not fully understood. Thus, we aimed to determine whether inactivating patterns of DNA-level alterations affect genes encoding for individual NRF2 inhibitor complex components (CUL3/KEAP1/RBX1) occur in PTC.

Findings: Combined patterns of epi/genetic alterations for KEAP1/CUL3/RBX1 E3 ubiquitin-ligase complex components were simultaneously interrogated for a panel of 310 PTC cases and 40 adjacent non-malignant tissues. Data were obtained from The Cancer Genome Atlas project. Enrichment of NRF2 pathway activation was assessed by gene-set enrichment analysis using transcriptome data. Our analyses revealed that PTC sustain a strikingly high frequency (80.6\%) of disruption to multiple component genes of the NRF2 inhibitor complex. Hypermethylation is the predominant inactivating mechanism primarily affecting KEAP1 (70.6\%) and CUL3 (20\%), while copy number loss mostly affects RBX1 (16.8\%). Concordantly, NRF2-associated gene expression signatures are positively and significantly enriched in PTC.

Conclusions: The KEAP1/CUL3/RBX1 E3-ubiquitin ligase complex is almost ubiquitously affected by multiple DNA-level mechanisms and downstream NRF2 pathway targets are activated in PTC. Given the importance of this pathway to normal thyroid function as well as to cancer; targeted inhibition of NRF2 regulators may impact strategies for therapeutic intervention involving this pathway.
\end{abstract}

Keywords: KEAP1/CUL3/RBX1 E3-ubiquitin ligase complex, Gene disruption, NRF2, Thyroid cancer

\section{Introduction}

Production of reactive oxidative species (ROS) by thyroid cells is essential to normal hormonogenesis and growth of the thyroid gland [1,2]. However, increased production of ROS is damaging to cells, and therefore pathways controlling cellular defensive mechanisms in response to ROS, such as the NFE2-related factor 2 (NRF2) pathway are of particular importance to the thyroid. Paradoxically, constitutive activation of this pathway occurs in several

\footnotetext{
*Correspondence: vmartinez@bccrc.ca

${ }^{\dagger}$ Equal contributors

BC Cancer Research Centre, BC Cancer Agency, 675 West 10th Avenue, Vancouver, BC V5Z1L3, Canada
}

tumor types affecting multiple oncogenic functions [3] (Figure 1A). Recently, the NRF2 pathway was found activated in papillary thyroid carcinoma (PTC) [4].

Activating mutations in the NRF2 gene, NFE2L2, are not common in PTC and genetic mechanisms underlying NRF2 activation remain to be elucidated for this tumor type [4]. NRF2 protein levels are negatively regulated by the KEAP1/CUL3/RBX1 E3 ubiquitin-ligase complex [5] (Figure 1A). Disruption of any complex component is sufficient to disrupt complex function and activate NRF2, highlighting the importance of simultaneously examining alterations to multiple complex components [6].

\section{Biomed Central}




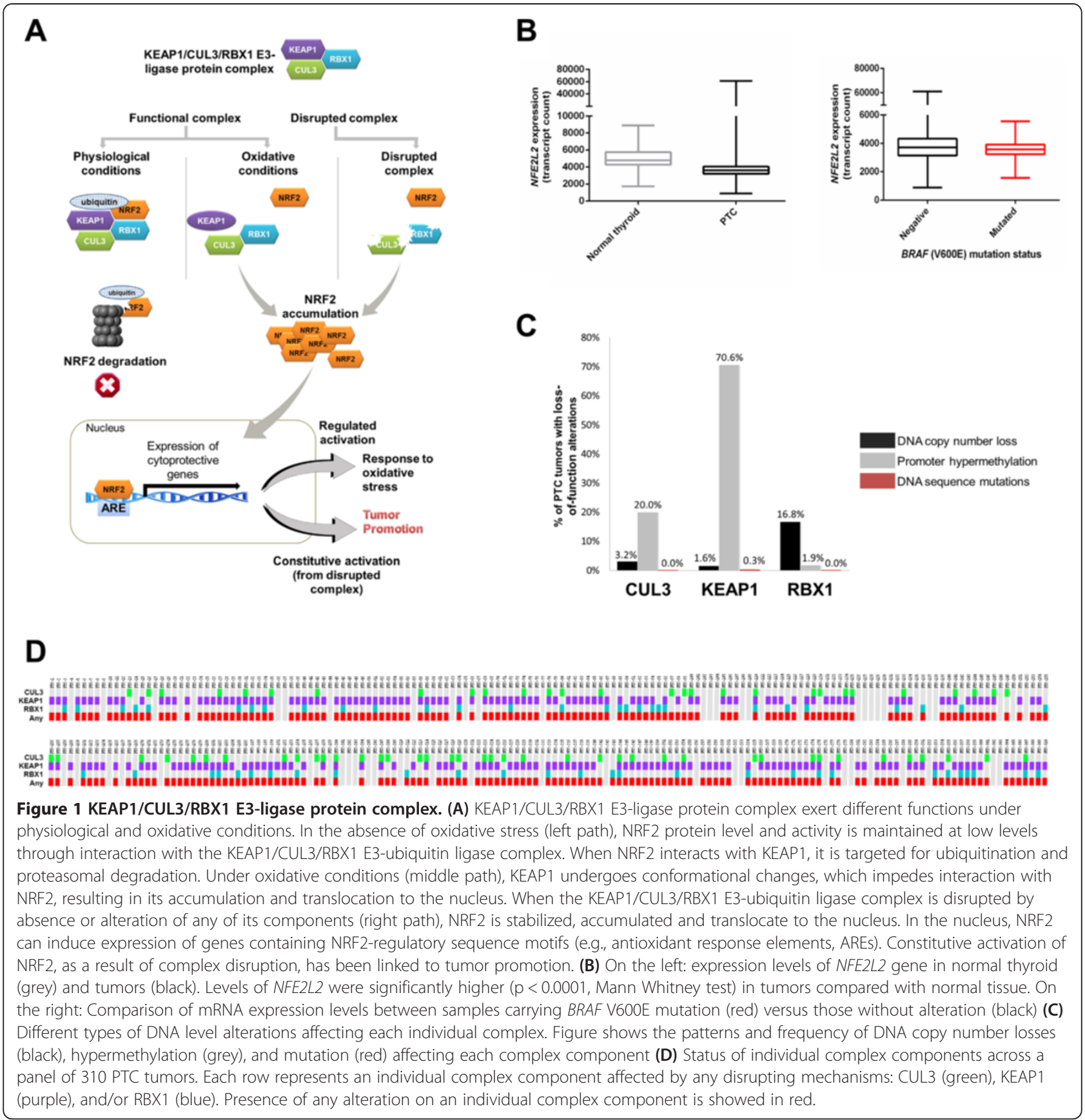

Since tumor genomes are disrupted by multiple genetic mechanisms, integration of multi-'omics data for individual tumors represents a powerful approach for discovering genes frequently altered. Therefore, we simultaneously investigated DNA sequence mutations, gene DNA copy number, and DNA methylation affecting key components of the KEAP1/CUL3/RBX1 complex and the NFE2L2 gene using a cohort of 310 PTC tumors from The Cancer Genome Atlas (TCGA) project (Additional file 1). We hypothesize that component gene disruption (DNA level) of the KEAP1/CUL3/RBX1 complex is a frequent event in PTC and may explain activation of NRF2 previously observed in PTC. We report a remarkably high frequency of DNA disruption to NRF2 inhibitor complex components and gene expression patterns in PTC tumors concordant with NRF2 activation.

\section{Analysis of mutations affecting NRF2 activation}

We first examined whether known mutational NFE2L2 events were frequent in the PTC cohort. No NFE2L2 mutations were detected, and only 1 out of 310 tumors (0.32\%) exhibited NFE2L2 copy number gains. NRF2 
protein overexpression has recently been described in PTC; however, protein information was not available for this cohort. Therefore, we assessed NFE2L2 mRNA expression in PTC tumors compared to non-malignant tissues, and found that underexpression of NFE2L2 in tumors reached statistical significance $(\mathrm{p}<0.0001)$ when compared to normal tissue (Figure 1B). Additionally, we examined 6,244 unique thyroid cancer tumors in the Catalogue of Somatic Mutations in Cancer (COSMIC). Of these, 128 (2.05\%) harbor KRAS mutations. Since regulation of NRF2 normally occurs through protein degradation, this mRNA finding was not surprising.

We were also interested in evaluating NFE2L2 mRNA expression in relation to $B R A F$ and $K R A S$ mutations, since mutations to these genes occur frequently in PTC [7], and mutated KRAS and BRAF are associated with increased transcription of NFE2L2 in other cancers [8]. Three PTC tumors $(<1 \%)$ exhibited mutations in $K R A S$ (affecting the Ras domain) whereas 177 (57.1\%) sustained BRAF mutations (predominantly V600E). Differential NFE2L2 mRNA expression between tumors with and without BRAF mutations was not evident (Figure 1B). Taken together, NRF2 pathway activation in PTC likely occurs through molecular mechanisms independent of those affecting NFE2L2, $B R A F$ or KRAS.

KEAP1/CUL3/RBX1 complex is frequently disrupted in PTC We next investigated inactivating DNA alterations affecting genes encoding the NRF2 inhibitor E3-ubiquitin ligase complex, specifically: KEAP1, CUL3 and RBX1. We calculated the frequency of copy number loss (CNL), promoter hypermethylation and/or mutations affecting these genes. Overall, frequencies of disruption were extraordinarily high and also varied amongst genes (KEAP1, 71.2\%; CUL3, 21.9\%; RBX1, 18.4\%). Hypermethylation was the main mechanism affecting KEAP1 (70.6\%) and CUL3 (20\%), while $R B X 1$ was almost exclusively affected by CNL (16.8\%) (Figure 1C, Additional file 2). Sequence mutations were not identified except for one KEAP1 G477S mutation (Figure 1C). Strikingly, when complex disruption was considered cumulatively, $80.6 \%$ of PTCs harbored a DNA alteration in at least one of the complex components (Figure 1D).

These findings indicate that multiple DNA mechanisms simultaneously affect different components of the KEAP1/CUL3/RBX1 complex at a very high frequency in PTC. Moreover, these results provide a plausible DNA level mechanism for recent findings by others describing NRF2 pathway activation in PTC [4].

\section{Multiple NRF2-related functions are significantly enriched among aberrantly expressed genes in PTC}

We next evaluated whether disruption to the NRF2 inhibitory complex in PTC was associated with increased transcription of NRF2 transcriptional targets (i.e. those containing the consensus NRF2-binding motif). Indeed, NRF2 target genes were significantly and positively enriched (nominal $\mathrm{p}$-value $=0.023$ ) in tumors relative to non-malignant thyroid tissues (Figure 2A).

Intriguingly, multiple gene sets associated with activated NRF2 were by far the most significantly enriched overall (FDR < 0.25) (Figure $2 \mathrm{~B})$. Notably, five independent gene sets corresponded to the well-known NRF2 activator, c-JUN [9] and two to the transcription factors BACH1 and $\mathrm{BACH} 2$. These findings are highly concordant with NRF2 pathway activation in PTC. Ranked genes corresponding to NRF2 transcriptional targets are presented in Figure 2C. The highest of these, ETV5, contains a consensus NRF2-binding motif and is also a target of thyroidspecific transcription factor FOXE1 [10]. FOXE1 was not overexpressed at the mRNA level in the PTC dataset (fold change $=0.77$ ).

\section{Discussion}

Abundance of physiological ROS results in oxidative stress which is damaging to cells and can lead to malignant transformation. Paradoxically, the NRF2 pathway, which is responsible for protecting cells against ROS damage, is constitutively activated in multiple cancer types and associated with many cancer hallmarks [11,12]. Overactivation of this pathway has recently been described in PTC [4]. Given recent findings and the importance of ROS to normal thyroid hormonogensis, elucidation of the molecular mechanisms underlying NRF2 deregulation in PTC is relevant. Here, we provide evidence of DNA mechanisms that likely promote stabilization and activation of NRF2 in PTC.

We answered questions pertaining to NRF2 activation utilizing a large panel of PTCs with multi-'omics data from the TCGA data portal. Consistent with previous reports, activating mutations or copy number gains/amplifications targeting NFE2L2 are uncommon in PTC [4]. Moreover, while BRAF V600E mutations were common, these were not associated with increased NRF2 expression in PTC, as described for other cancers [8], indicating that NRF2 pathway activation in PTC likely occurs through other mechanisms (Figure 1B).

The KEAP1/CUL3/RBX1 complex is a key negative regulator of NRF2. We applied a multi'omics approach to interrogate DNA disruptions to KEAP1/CUL3/RBX1 complex components (Figure 1A). In a previous study, Ziros et al., found no correlation between KEAP1 and NRF2 protein expression levels, arguing against decreased KEAP1 levels as mechanisms for NRF2 activation [4]. Based on our results, alterations affecting other complex components, such as CUL3 and RBX1, might be also contributing to increased expression of NRF2 observed in PTC. We found that DNA copy number loss 


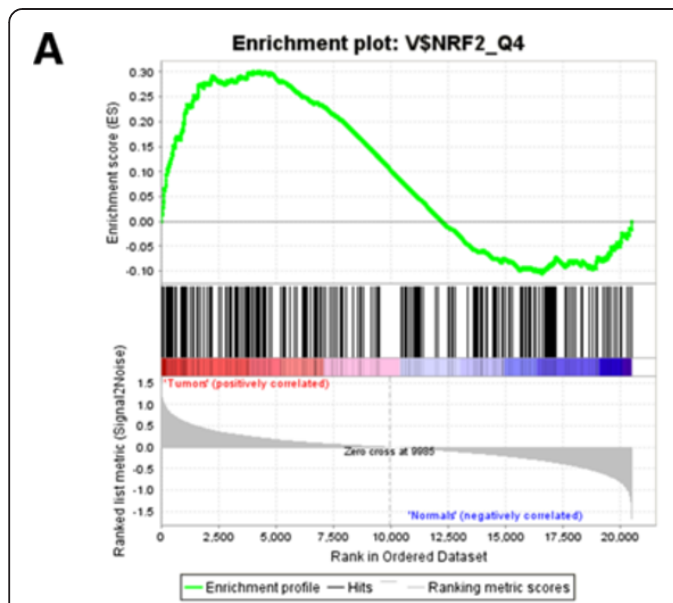

B

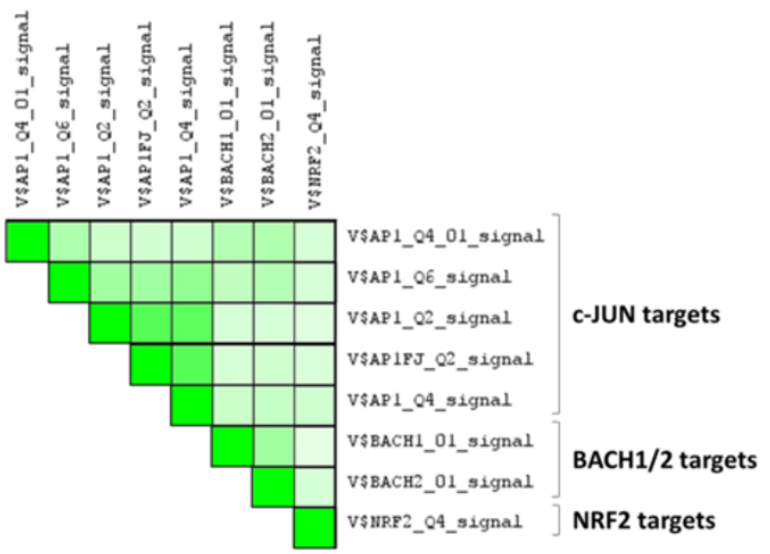

C

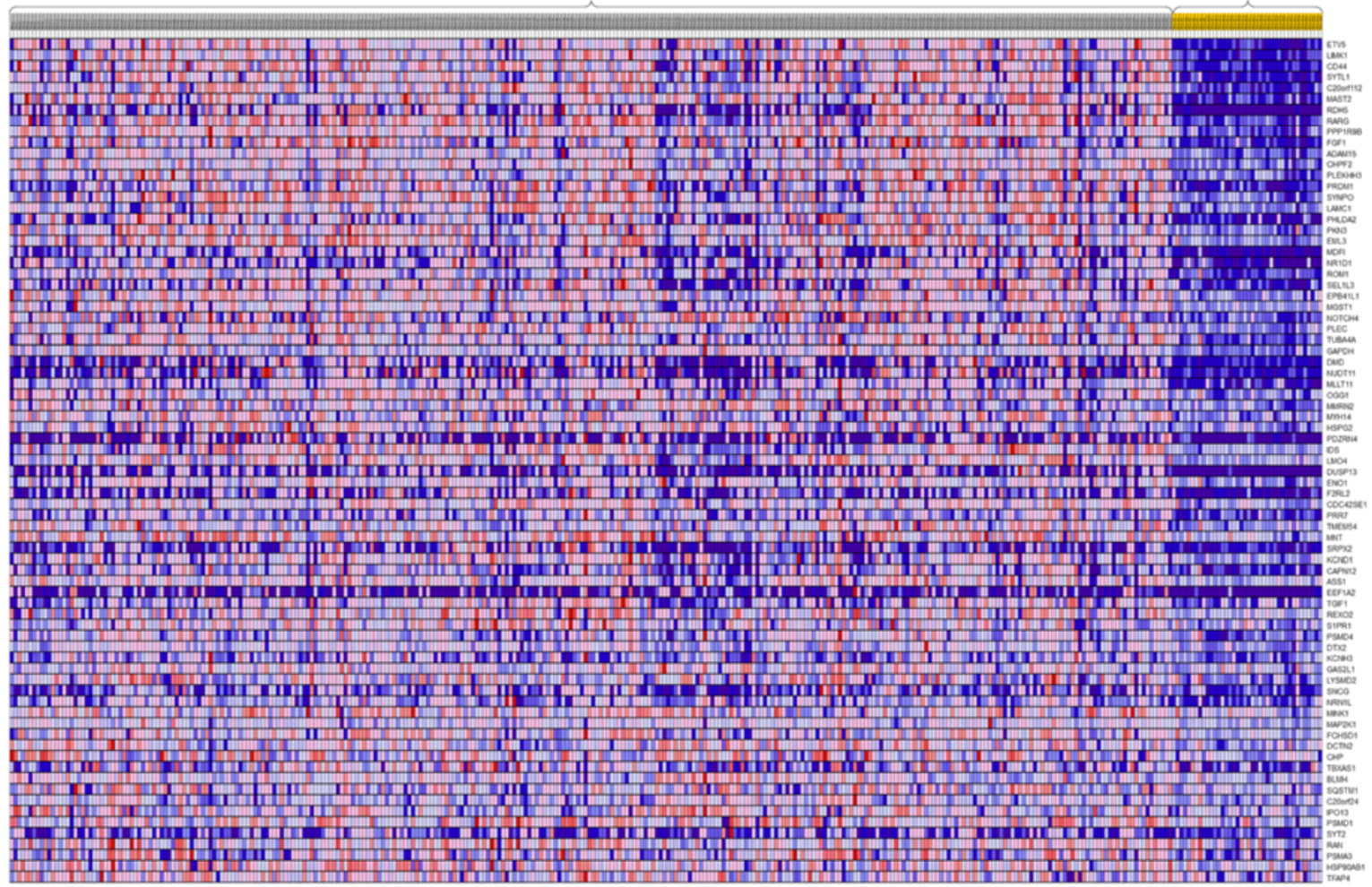

Figure 2 PTC tumors are significantly and positively enriched for gene sets corresponding NRF2 related functions. (A) A gene set (V\$NRF2_Q4) consisting of NFE2L2 transcriptional targets was significantly and positively enriched in PTC tumor relative to non-malignant tissues. For PTC tumors, normalized enrichment score $(E S)=1.42$, p-value $=0.023$. ES (green line) represent the probability that the gene set is positively (left side) or negatively (right side) enriched in a ranked gene list. Genes are first ranked based on differences between tumor and normal groups. Ranked genes that appear in the defined gene set are indicated as "Hits" below the Enrichment profile (black hash marks). Genes ranked near the top of the gene list are underscored with a red bar, whereas genes near the bottom of the list are underscored with a blue bar. (B) A Leading Edge Analysis comparing whether highly ranked genes corresponding to different gene sets overlapped. Highest ranked genes refer to those most highly differentially expressed genes between tumors compared to normals. Leading edge genes (i.e. those most different at level of expression between PTC tumors and normals) that correspond to highly statistically significantly enriched gene sets overlap with NRF2 transcriptional targets. These gene sets corresponded to functions related to NRF2, such as C-JUN (AP1 gene sets), a well-known transcriptional activator of NFE2L2, and BACH1 and 2, which are transcriptional repressors that compete with NRF2 to bind ARE. 100\% and 0\% overlap in leading edge genes are indicated by dark green and white, respectively. (C) A gene expression heatmap was generated for NRF2 transcriptional target genes that are over-expressed in PTC tumors relative to normals. Expression values for each gene are represented as colors, where the range of colors (red, pink, light blue, dark blue) shows the range of expression values (high, moderate, low, lowest). 
and promoter hypermethylation frequently affect individual components, that when considered together affect a remarkably high proportion of PTCs (80\%). This phenomenon has been observed by our group and others in lung cancer $[6,13]$. However, the frequency by which multiple complex components were simultaneously disrupted, and the molecular mechanisms affecting individual components, were strikingly different for PTC (Figure 1C-D), compared to other tumor types.

Promoter hypermethylation is the predominant mechanism affecting KEAP1 and CUL3 in PTC. Silencing of KEAP1 by hypermethylation has been described in lung cancer [14], and associated with stabilized NRF2 and increased expression of NRF2 target genes in colorectal cancer [15]. While KEAP1 mutations are frequent in other cancers, they are not in PTC. However, germ-line KEAP1 mutations have been identified in a family with multinodular goiter [16], alluding to the importance of this gene to normal thyroid physiology. Interestingly, segmental DNA losses is almost the exclusively the type of alteration affecting $R B X 1$. This phenomenon has been also observed at a similar frequency in lung tumors, with a concurrent complex function disruption [6].

Gene set enrichment analysis (GSEA) revealed that not only were genes containing the consensus NRF2binding motif over-expressed in PTCs, but overall, the most significantly enriched gene sets corresponded to functions related to NRF2, including the NRF2 activator c-JUN and the NRF2 target competitive binders, BACH1 and 2 (Figure 2B). Bach1, competes with NRF2 protein for binding to target genes, leading to inhibited expression of NQO1 and HO-1 for example [17-19]. These results indicate that in addition to DNA disruption to NRF2 complex inhibitors, the NRF2 pathway is also likely activated by increased activity of NRF2 activators and decreased activity of NRF2 gene target transcriptional repressors in PTCs.

ETV5 was the putative NRF2 transcriptional target most highly upregulated in tumors compared to controls. ETV5 is an oncogenic member of the ETS transcription factor family, associated with cell proliferation and metastasis in various cancers [20], and a known target of thyroid-specific transcription factor FOXE1. Although we did not detect overexpression of FOXE1 in PTCs at the mRNA level, further exploration of ETV5 in the context of NRF2 signaling is warranted.

Taken together, we found that i) DNA level disruption of NRF2 inhibitory complex components occurs at an extremely high frequency in PTC, ii) primarily by promoter hypermethylation, iii) NRF2 transcriptional targets are overexpressed in PTC, and iv) activation of NRF2 in PTC is complex and likely involves activation and inhibition of multiple NRF2 activators and repressors.
A major caveat of this study is the lack of protein assessment. However, the data presented here provide rationale to interrogate the role of the NRF2 pathway in PTC at the protein level, and elucidate the target molecules driving PTC phenotypes. In summary, we present compelling evidence that activation of the NRF2 pathway is extraordinarily, selectively activated in PTC and provide further rationale for exploration of this pathway as a therapeutic target in PTC.

\section{Additional files}

\begin{abstract}
Additional file 1: Supplementary Methods.
Additional file 2: Figure S1. DNA copy number alterations affecting components of the KEAP1/CUL3/RBX1 complex. DNA copy number affecting KEAP1/CUL3/RBX1 complex components in the TCGA PTC set. Copy number is estimated based on probe intensity derived from the Genome-Wide Human SNP Array 6.0 (Affymetrix). Around 17\% of samples exhibit copy number losses (in blue, see Figure legend) affecting RBX 1 gene. This appears to be a particular mechanism for gene disruption affecting this complex component, since the rest of complex components exhibit alterations at copy number level in a considerably lower frequency of samples.
\end{abstract}

Competing interests

The authors have no competing interests to declare.

\section{Authors' contributions}

VDM and EAV contributed equally in study design, data analysis and manuscript writing. $L A P, R H, K L T, W L L$ contributed to data interpretation and manuscript revision. All authors read and approved the final manuscript.

\section{Acknowledgements}

Funding from Canadian Institutes for Health Research (CIHR) and National Institutes of Health (NIH/NCI) 1R01CA164783-01, and scholarships from University of British Columbia, Vanier Canada, and CIHR.

Received: 29 July 2013 Accepted: 2 October 2013

Published: 20 October 2013

\section{References}

1. Poncin S, Van Eeckoudt S, Humblet K, Colin IM, Gerard AC: Oxidative stress: a required condition for thyroid cell proliferation. Am J Pathol 2010, 176:1355-1363.

2. Poncin S, Colin IM, Gerard AC: Minimal oxidative load: a prerequisite for thyroid cell function. J Endocrinol 2009, 201:161-167.

3. Ganan-Gomez I, Wei Y, Yang H, Boyano-Adanez MC, Garcia-Manero G: Oncogenic functions of the transcription factor Nrf2. Free Radic Biol Med 2013, 65:750.

4. Ziros PG, Manolakou SD, Habeos IG, Lilis I, Chartoumpekis DV, Koika V, Soares P, Kyriazopoulou VE, Scopa CD, Papachristou DJ, Sykiotis GP: Nrf2 Is commonly activated in papillary thyroid carcinoma, and it controls antioxidant transcriptional responses and viability of cancer cells. $J$ Clin Endocrinol Metab 2013, 98(8):E1422.

5. Cullinan SB, Gordan JD, Jin J, Harper JW, Diehl JA: The Keap1-BTB protein is an adaptor that bridges Nrf2 to a Cul3-based E3 ligase: oxidative stress sensing by a Cul3-Keap1 ligase. Mol Cell Biol 2004, 24:8477-8486.

6. Thu KL, Pikor LA, Chari R, Wilson IM, Macaulay CE, English JC, Tsao MS, Gazdar AF, Lam S, Lam WL, Lockwood WW: Genetic disruption of KEAP1/ CUL3 E3 ubiquitin ligase complex components is a key mechanism of NF-kappaB pathway activation in lung cancer. J Thorac Oncol 2011, 6:1521-1529.

7. Caronia LM, Phay JE, Shah MH: Role of BRAF in thyroid oncogenesis. FASEB J 2011, 17:7511-7517.

8. DeNicola GM, Karreth FA, Humpton TJ, Gopinathan A, Wei C, Frese K, Mangal D, Yu KH, Yeo CJ, Calhoun ES, et al: Oncogene-induced Nrf2 
transcription promotes ROS detoxification and tumorigenesis. Nature 2011, 475:106-109.

9. Venugopal R, Jaiswal AK: Nrf2 And Nrf1 in association with Jun proteins regulate antioxidant response element-mediated expression and coordinated induction of genes encoding detoxifying enzymes. Oncogene 1998, 17:3145-3156.

10. Fernandez LP, Lopez-Marquez A, Martinez AM, Gomez-Lopez G, Santisteban $P$ : New insights into FoxE1 functions: identification of direct FoxE1 targets in thyroid cells. PLoS One 2013, 8:e62849.

11. Shelton $P$, Jaiswal AK: The transcription factor NF-E2-related factor 2 (Nrf2): a protooncogene? FASEB J 2013, 27:414-423.

12. Muller T, Hengstermann A: Nrf2: friend and foe in preventing cigarette smoking-dependent lung disease. Chem Res Toxicol 2012, 25:1805-1824.

13. Lee DF, Kuo HP, Liu M, Chou CK, Xia W, Du Y, Shen J, Chen CT, Huo L, Hsu MC, et al: KEAP1 E3 Ligase-mediated downregulation of NF-kappaB signaling by targeting IKKbeta. Molecular cell 2009, 36:131-140.

14. Guo D, Wu B, Yan J, Li X, Sun H, Zhou D: A possible gene silencing mechanism: hypermethylation of the Keap1 promoter abrogates binding of the transcription factor Sp1 in lung cancer cells. Biochem Biophys Res Commun 2012, 428:80-85.

15. Hanada N, Takahata T, Zhou Q, Ye X, Sun R, Itoh J, Ishiguro A, Kijima H, Mimura J, Itoh K, et al: Methylation of the KEAP1 gene promoter region in human colorectal cancer. BMC Cancer 2012, 12:66.

16. Teshiba R, Tajiri T, Sumitomo K, Masumoto K, Taguchi T, Yamamoto K: Identification of a KEAP1 germline mutation in a family with multinodular goitre. PLoS One 2013, 8:e65141.

17. Liu D, Duan X, Dong D, Bai C, Li X, Sun G, Li B: Activation of the nrf2 pathway by inorganic arsenic in human hepatocytes and the role of transcriptional repressor bach1. Oxid Med Cell Longev 2013, 2013:984546.

18. Sun J, Hoshino H, Takaku K, Nakajima O, Muto A, Suzuki H, Tashiro S, Takahashi S, Shibahara S, Alam J, et al: Hemoprotein Bach1 regulates enhancer availability of heme oxygenase-1 gene. EMBO J 2002, 21:5216-5224.

19. Dhakshinamoorthy S, Jain AK, Bloom DA, Jaiswal AK: Bach1 Competes with $\mathrm{Nrf} 2$ leading to negative regulation of the antioxidant response element (ARE)-mediated NAD(P)H: quinone oxidoreductase 1 gene expression and induction in response to antioxidants. J Biol Chem 2005, 280:16891-16900.

20. Oh S, Shin S, Janknecht R: ETV1, 4 And 5: an oncogenic subfamily of ETS transcription factors. Biochim Biophys Acta 1826, 2012:1-12.

doi:10.1186/1476-4598-12-124

Cite this article as: Martinez et al:: Frequent concerted genetic

mechanisms disrupt multiple components of the NRF2 inhibitor KEAP1/

CUL3/RBX1 E3-ubiquitin ligase complex in thyroid cancer. Molecular

Cancer 2013 12:124.

\section{Submit your next manuscript to BioMed Central and take full advantage of:}

- Convenient online submission

- Thorough peer review

- No space constraints or color figure charges

- Immediate publication on acceptance

- Inclusion in PubMed, CAS, Scopus and Google Scholar

- Research which is freely available for redistribution 